\title{
Positive Animal Welfare: Bridging the Gap or Raising Inequalities Worldwide?
}

\author{
Jean-Loup Rault ${ }^{1 *}$, Peter Sandøe ${ }^{2}$, Quixi Sonntag ${ }^{3}$ and Leopoldo Stuardo ${ }^{4}$ \\ ${ }^{1}$ Institute of Animal Welfare Science, University of Veterinary Medicine, Vienna, Austria, ${ }^{2}$ Department of Food and Resource \\ Economics and Department of Veterinary and Animal Sciences, University of Copenhagen, Copenhagen, Denmark, \\ ${ }^{3}$ Department of Production Animal Studies, Faculty of Veterinary Science, University of Pretoria, Pretoria, South Africa, \\ ${ }^{4}$ Standards Department, World Organisation for Animal Health, Paris, France
}

\section{OPEN ACCESS}

Edited by: Janice Swanson,

Michigan State University,

United States

Reviewed by:

Siobhan Mullan

University College Dublin, Ireland

*Correspondence:

Jean-Loup Rault

jean-loup.rault@vetmeduni.ac.at

Specialty section:

This article was submitted to Animal Welfare and Policy,

a section of the journal

Frontiers in Animal Science

Received: 30 November 2021

Accepted: 24 January 2022

Published: 15 February 2022

Citation:

Rault J-L, Sandøe P, Sonntag Q and

Stuardo L (2022) Positive Animal

Welfare: Bridging the Gap or Raising Inequalities Worldwide?

Front. Anim. Sci. 3:825379.

doi: 10.3389/fanim.2022.825379
Positive animal welfare (PAW) is a rising topic in animal welfare science, although its construct, definition, and operational approach remain debated. Despite this scientific uncertainty, there is societal interest to include more indicators of positive welfare in legislation, animal welfare assessment and accreditation schemes. Changes in some farming practices seem to be in line with promoting PAW (e.g., free-range housing), providing animals more opportunities for positive experiences such as rewarding natural behaviour, greater autonomy, or choice. Interestingly, some of the ideas underlying PAW are present in extensive production systems or low-input animal management practices that are common in low-income countries, for example free-roaming livestock or village dogs. Nevertheless, welfare challenges such as neglect, diseases, poor nutrition, animal abuse and other forms of suffering remain ubiquitous, especially where resources like veterinary support are limited. Living conditions for animals in low-income countries provide examples of the delicate balance between positive welfare and welfare risks relating to health and survival, with inextricable ethical dilemmas. In our view, the growing focus on PAW could stimulate a more balanced approach to animal welfare worldwide, promoting PAW while simultaneously limiting various forms of welfare challenges. However, this requires accounting for human factors such as societal and cultural location-specific aspects to find flexible solutions that also benefit and respect people whose livelihood may be at stake. Those human factors also modulate the consideration and importance of providing animals with positive welfare states and the role of underlying ethical concepts like happiness and "a good life."

\footnotetext{
Keywords: developing countries, human behaviour, low-income countries, one health, positive welfare, well-being, one welfare
}

\section{THE RISE OF POSITIVE WELFARE}

The term "positive animal welfare" may, for someone naive to the development of animal welfare science, come across as redundant: According to the Cambridge Advanced Learner's Dictionary and Thesaurus the word "welfare" has as one of its core meanings "physical and mental health and happiness", hence welfare by definition encompasses positive welfare.

However, in animal welfare science the meaning of the term "welfare" has been transformed so that it is understood as a state of an animal that can range from poor to good (Broom, 1991). 
Historically, in the context of efforts to secure animal welfare, the focus has then been on avoiding poor welfare or "welfare challenges" (eSandoe and Jensen, 2013). Welfare challenges can occur as various types and in various degrees, with at its extreme suffering (Olsson et al., 2019). Following this, a large part of animal welfare science that developed from the 1970s onwards focussed on measuring signs of physical and behavioural needs of animals not being fulfilled, i.e., on mortality, morbidity or signs of frustration. Hence, in practice animal welfare came to mean that animals survived, did not get ill and did not show signs of frustration.

The recent interest in positive animal welfare may partly be understood in the light of this situation. Inspired by a zeitgeist occupied with human flourishing and positive psychology (Seligman and Csikszentmihalyi, 2000), those concerned with how animals are treated have come to think that there must be more to animal welfare than just the absence of sufferinganimals should not just survive and be healthy, they should flourish and be happy (Yeates and Main, 2008; Lawrence et al., 2019; Webb et al., 2019; Rault et al., 2020; Vigors et al., 2021), or have what they want (Dawkins, 2021). The interest in positive animal welfare has also been partly inspired by developments within animal welfare science. Scientists working on the behavioural needs of animals have increasingly focused on the positive side of the equation with various attempts to conceptualise the idea of enabling species-specific behaviour and natural living (Lawrence et al., 2019). There has also been a growing interest in understanding play and other signs of positive mental states in animals (Boissy et al., 2007). Finally, there have been researchers developing ideas of positive welfare in terms of animals being occupied (Mellor, 2015) or animals being in charge or exercising autonomy (Spinka, 2019).

These new societal and scientific developments regarding positive animal welfare are clearly originating from the highincome and Western parts of the world. It may be tempting to see them as diversion from the efforts to ensure that basic welfare requirements are fulfilled for all animals in human care and in all parts of the world, with a focus on avoiding a wide range of unpleasant mental states as welfare challenges (e.g., the Five Freedoms approach; British Farm Animal Welfare Council, 1993). However, it may also be possible to turn the idea around and view positive welfare as an opportunity for those living in the less well-off parts of the world where animals are often kept in more extensive ways with better opportunities to exercise species-specific behaviour, autonomy and other aspects of what is considered positive welfare. In this paper, we will take a comparative approach to the ways animals are kept in various parts of the world, and highlight the opportunities and potential barriers to promote positive animal welfare in a global context.

\section{ANIMAL MANAGEMENT AND HUSBANDRY: DIFFERENT CHALLENGES, UNIVERSAL NEEDS}

Positive welfare is currently not a unified concept (Lawrence et al., 2019; Rault et al., 2020), but inferences can be drawn from recent examples in animal management or husbandry. The ability for animals to express normal behaviour (Fraser, 2008), and being engaged with a perception of control over their environment (Mellor, 2015), resonate with consumers and Western societal views in general (Lassen et al., 2006; Carnovale et al., 2021), leading to a rising interest for "welfarefriendly" farming systems in high-income countries where the products are sold with special labels (Heerwagen et al., 2015). Those housing conditions typically provide animals with more behavioural opportunities, such as greater freedom of movement, outdoor access, and generally more complex environments; hence with greater potential to trigger positive experiences and thereby positive welfare. However, those housing systems can also come with greater risks, such as potentially higher mortality or disease incidence (Lay et al., 2011; Weeks et al., 2016; Hemsworth, 2018), due to the same provisions that in other circumstances or for other individuals can elicit positive welfare from the opportunities mentioned above.

Animals in low-income countries often live in conditions that one could consider similar to those "welfare-friendly" systems, where they are free to roam around, forage and express an array of natural behaviours. This is not only the case for farm animals. In fact, free-roaming dogs (owned or not) are estimated to constitute approximately $75 \%$ of the dog population worldwide (> 700 million to $\sim 1$ billion, Lord et al., 2013), making freeroaming dogs the norm and "pet" dogs the exception (see Box 1). Although free-roaming animals are potentially able to meet some of their own needs, good welfare does not necessarily arise from total freedom and no human intervention, i.e., animals left to their own. Mortality and morbidity, indicative of serious animal welfare challenges, are still prevalent in many parts of the world. For example, $81 \%$ of free-roaming dogs in a studied population in India died before 7 months of age, and $63 \%$ of that mortality was considered to be due to humans (Paul et al., 2016). In Africa, diseases, predation and drought have been estimated to cause the death of one in four young ruminants and one in 10 adult ruminants each year (Grace et al., 2012).

Animals from the same species have universal needs wherever they live in the world, shaped by evolution into species-specific needs. Those animal needs are to a large degree retained in domestic animals (Price, xbib1999), irrespective of housing and management systems, genetic selection, living environments, or their utility to humans. This offers the advantage that we can use a common basis to assess animal welfare states, such as with animal-based measures, i.e., indicators of how the animal copes with the conditions in which it lives (e.g., body condition, cleanliness, disease or injury, gait, but also social interactions, play and other behaviours; Butterworth et al., 2018; and see below last section).

Despite the rising scientific interest for positive welfare, there are still currently few validated indicators of positive welfare

\footnotetext{
${ }^{1}$ We use 'pet' here in the general sense of the term of an animal cared for by a specific individual for the purpose of companionship. What people consider a 'pet' may differ between cultures and definitions, as some people may consider for example a free-roaming animal their pet, and other animals may be cared for by the community rather than an individual person.
} 


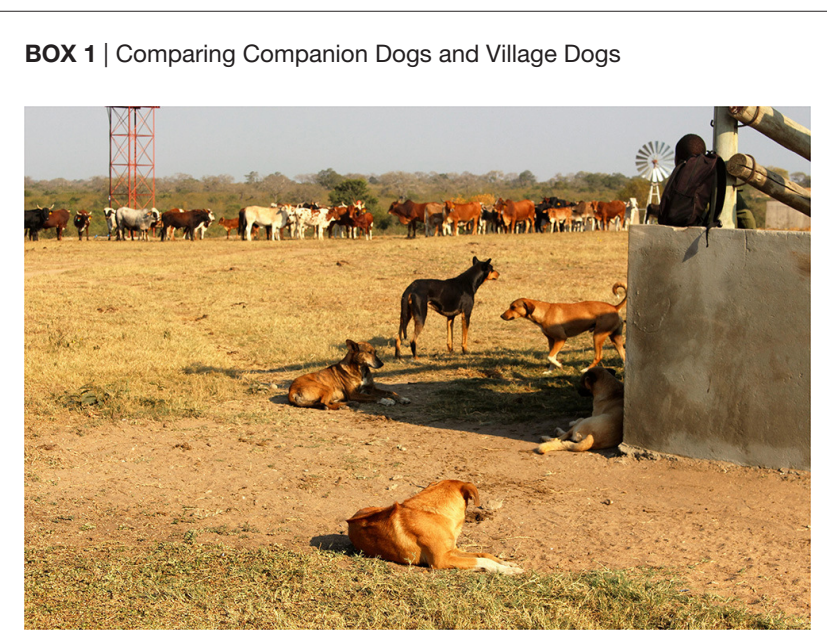

The World's dog population has been estimated to consist of between 500 million and 1 billion individuals. Of these only a minority, about 150 million in the United States and Europe and a smaller number of millions in other parts of the world, live as companion dogs (Lord et al., 2013). Some of the remaining dogs are working dogs, but the vast majority are free-roaming dogs in different parts of the world; and many of these are so-called "village dogs." Comparing companion dogs and village dogs, it is obvious that the village dogs have an advantage when it comes to positive welfare. In many ways village dogs live a 'natural' life in line with what they have adapted for during the last 10,000-15,000 years where they have existed as a species. They live close to humans, typically associating with one or more human families but also with opportunities to roam, dig and scavenge and to seek other canine and human company. Therefore, they are occupied and can exercise a large degree of autonomy. Unlike these, many dogs living as companion animals in modern, urban or suburban settings live lives where they, to a large degree, miss out on these aspects of positive welfare. Companion dogs typically are much more confined and many of them have to manage loneliness most (human) working days, which in turn gives rise to a number of behavioural problems that negatively affect their welfare (Amat et al., 2014). However, when it comes to health-related welfare challenges the typical villages dog is likely to be more affected than the companion dog by under- or malnutrition, lack of veterinary care, parasites and infectious diseases, and hostility from humans. These problems are typically aggravated by uncontrolled reproduction. Consequently, free-roaming dogs face a very high level of pup mortality, with a life expectancy less than a year, much lower than the 10 to 12 years found among companion dogs (Paul et al., 2016). Picture source: Q. Sonntag.

to allow its assessment in practice (Boissy et al., 2007; Yeates and Main, 2008; Keeling et al., 2021). While there has been a lot of research on practices or situations that induce animal suffering (Dawkins, 2008), the way positive experiences can buffer or alleviate negative welfare experiences and improve the resulting welfare state of an animal remains scientifically poorly understood (Yeates and Main, 2008; Fife-Cook and Franks, 2019; Lawrence et al., 2019; Rault et al., 2020). Finding valid and robust indicators of positive welfare should help to understand its contribution to animal welfare states, and these indicators could potentially be integrated to animal welfare assessment schemes worldwide.

Thus, high- and low-income countries alike may be able to adopt common approaches (e.g., policies, assessment) that comprehensively address animal welfare by avoiding welfare challenges while promoting opportunities for positive experiences.

\section{POSITIVE WELFARE: A FIRST WORLD PROBLEM OR AN OPPORTUNITY FOR LOW-INCOME COUNTRIES?}

The historical approach to animal welfare in Western culture evolved over time from emphasising the need to reduce welfare challenges to the need to ensure positive welfare (Lawrence et al., 2019). The question arises whether this sequence is the necessary route to follow for other parts of the world, or whether positive animal welfare can be attained without first addressing the need to reduce welfare challenges? To answer this question, three factors need to be considered: the salience of animal welfare in a given society; the cultural, religious and social diversity of communities interacting with domestic animals; and the way people value animals.

The salience of animal welfare refers to the importance people attach to it. Historically, much animal welfare change in highincome countries was driven by consumer opposition to industry practices that result in poor animal welfare (e.g., Harrison, 1964). Public pressure resulted in laws and regulations to be enacted (e.g., UK Brambell committee; Farm Animal Welfare British Farm Animal Welfare Council, 1993), or now changes driven by the market rather than regulation (Sandøe et al., 2020). Activism to promote animal welfare has become a salient issue for societies in high-income countries of the Western world. In low-income countries where people may be struggling to satisfy their most basic needs for survival, animal welfare often is not a priority due to its low salience in the presence of other more pressing issues. The importance of animal welfare relative to other more proximal issues is therefore often limited in resourcepoor communities.

In addition to the relative importance of animal welfare in a given society, cultural factors impact the way animal welfare is approached in different countries. Animals in Africa, for example, fulfil important roles linked to traditional healing practices, connecting with the ancestors and celebrations (Qekwana et al., 2019). Cultural beliefs around animals represent the world in a different manner to what is considered to be in line with findings from animal welfare science in Western terms, and may present obstacles when attempts are made to address animal health and welfare issues. However, the Western individualistic approach differs from African "ubuntu" culture, a worldview that espouses the values of sharing, participation, caring and mutual respect as expressed in the phrase "I am because you are, and you are because we are" (Nkondo, 2007). This collective approach to maintaining a cohesive society that includes respecting nature and animals could be helpful in employing more culturally sensitive approaches in dealing with societal change (Etiyibo, 2017).

These first two factors, namely the lack of salience of animal welfare and the cultural differences, do not imply that people with different priorities or beliefs cannot be sensitised to the needs of animals and change their behaviour toward animals. The solution 
BOX 2 | Case Study With a Community-Based, Participatory and Culturally Sensitive Approach

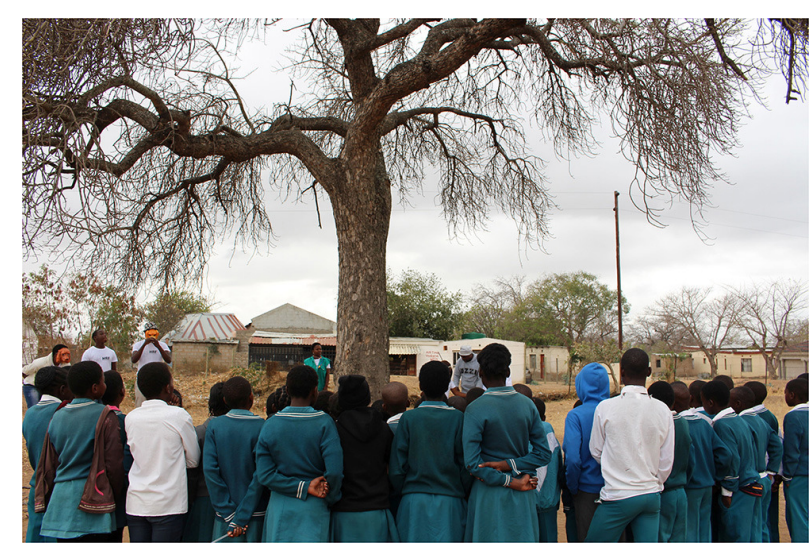

A study in a rural area in South Africa (Sonntag, 2018) investigated the utilisation of participatory reflection and action in facilitating awareness of rabies prevention. Participatory engagement employs an asset-based (positive psychology) approach (Whiting et al., 2012), emphasising the assets already present in a community and using these in an empowerment process. The participatory process furthermore encourages learning about and respect for local beliefs in co-creating solutions with community participants (Amauchi et al., 2021). In this case, a series of participatory meetings spanning several months enabled a group of community members to expand their knowledge about rabies and its prevention to the degree that they became local rabies experts. They were able to devise their own plan for rabies control and eventually took ownership of the emerging rabies challenge in their community. The participants utilised house-to-house information-sharing visits to engage with community members about rabies and produced an educational drama about rabies control that was performed at local primary schools, as depicted on the picture. Outcomes of the participatory process included increased rabies awareness in the community as well as a positive (and unexpected) change in attitudes towards dogs. This attitudinal change resulted in increased empathy towards dogs, thus supporting animal welfare. Picture source: Q. Sonntag.

may be in the way the topic of animal welfare is introduced or discussed. A community-based, participatory and culturally sensitive approach can be an effective way to change thinking and human behaviour related to animal welfare (Sonntag, 2018; see Box 2). Given this type of approach, it may be possible to draw upon existing values within a community to address positive animal welfare while simultaneously eliminating welfare challenges. Therefore, participative and inclusive approaches such as community-based initiatives can empower people to make a difference.

The third factor, namely the way people perceive the value of animals, drives animal welfare in different ways. When animals have a perceived economic value and improving their welfare leads to an increased profit, better welfare can be linked to a tangible positive consequence (McInerney, 2004). On the other hand, recognition of the inherent value of animals that places a moral responsibility on people to ensure that animals do not suffer, or at least do not suffer unnecessarily, can also improve animal welfare (Spooner et al., 2014). In Mexico, Portugal and
Spain, for example, the welfare of working equids was higher when their owners had an intrinsic appreciation of the animals as opposed to merely valuing them for their instrumental value (Proops et al., 2021). Consequently, both the instrumental value and intrinsic value of animals can drive change in practices that affect animal welfare.

It is already evident in low-income countries that the economic advantage of improving animal welfare can result in positive changes for animals. In Southern Africa, for example, animals destined for international trade experience better welfare in export abattoirs, as compared to animals in local abattoirs or during traditional slaughter at homesteads (Ndou et al., 2011; Qekwana et al., 2019). This phenomenon appears to be merely a response to the economic incentive and not necessarily a result of transformational thinking about the moral status of animals in terms of possessing intrinsic value. When animals do not necessarily have a practical value, understanding their intrinsic value may be addressed by building upon existing value systems such as ubuntu instead of introducing an unfamiliar Western point of view (Nkondo, 2007).

As it appears that minimum standards can be complied with when there is adequate perceived economic benefit to do so, one may assume that improvement in animal welfare can occur through the application of policies and regulations, driven by the perceived economic benefit. However, to achieve a more lasting change in attitudes toward animals, it would be necessary to evoke the ethical argument for the improvement of animal welfare. The duty of care, as an ethical consideration, may vary depending on cultural factors, and for instance whether someone feels responsible for that particular individual animal. It could be argued that when the ethical driver is successful in changing perceptions about animals, it could lead directly to the understanding that animals require optimal well-being. Thus, it might be possible to attain positive animal welfare directly, without first focussing solely on the minimisation of welfare challenges. Nevertheless, high-income countries have more access to resources and thus may be in a better position than low-income countries to address this trade-off between output efficiency and animal welfare.

\section{ACKNOWLEDGING ANIMAL WELFARE AS A MULTI-STAKEHOLDER TOPIC AND ITS DIVERSITY}

It is evident that the understanding and importance of animal welfare differs for each and every individual, region and culture. Animal welfare is a complex and multifaceted topic, involving scientific, ethical, economic, cultural, social, religious, and political dimensions (British Farm Animal Welfare Council, 1993; Fraser, 2008), and also intrinsically linked with the environment and human health (Pinillos, 2018).

Although livestock farms are getting larger and more concentrated in both high-income and low-income countries, 
the majority of livestock worldwide, primarily living in lowincome countries, are still kept in small scale farms, free-range or backyards (International Fund for Agricultural Development, 2013). However, it should be emphasised that small scale farming does not necessarily mean higher welfare, non-confined systems or greater free-roaming opportunities. Livestock make a significant contribution to the livelihoods of smallholder farmers in low-income countries, mainly through subsistence and small-scale commercial production. In Bangladesh for example, the farming system is dominated by smallholders $(96 \%$ of holdings) with diversified on-farm and off-farm livelihood strategies: most keeping livestock, tending homestead gardens, and cultivating rice (Perry et al., 2018). In Zambia, $82 \%$ of cattle, $97 \%$ of goats and $64 \%$ of sheep, respectively, are farmed on smallholdings (Zambia Ministry of Fisheries Livestock, 2019). This means that it can be challenging to reach the people caring for those animals, for example to raise awareness of the animals' needs (Braae et al., 2016). In addition, small scale farming typically present highly heterogeneous conditions, which requires taking into consideration the diversity of situations and means available.

Applied research plays a key role, particularly as it relates to indicators and ways of assessing animal welfare. The use of animal-based measures (also called performance or output criteria), describe welfare in terms of the animal's state, rather than the potential of the situation or what the animal is provided with. Animal-based measures are increasingly used to assess animal welfare, and a number of scientifically valid indicators are available (e.g., Butterworth et al., 2018; OIE, 2018). Therefore, where there are multifactorial problems and it is therefore difficult to provide specifications (resourcebased criteria) for all contributing factors, or when conditions are too heterogeneous (e.g., large differences between animalholding systems, countries, etc.), the most practical solution is to monitor animal-based criteria to ascertain the animal's welfare state. This approach can be efficient to tackle simultaneously welfare challenges and positive animal welfare in low- and middle-income countries and where resources are limited. Nevertheless, resource- and management-based measures can be valuable, when the resource has a strong predictive link to its welfare implication, or when expertise in animal welfare assessment or access to the animals is limited such as in the small-scale farming highlighted above and as an initial step toward progress.

However, focus on improving animal welfare requires an effort from the relevant stakeholders to educate not only farmers or caretakers but potentially also the competent authorities, possibly through improvement in the quality of the regulatory framework. To achieve consensus in ensuring animal welfare worldwide, the World Organisation for Animal Health (OIE) for example has since 2002 included animal welfare into its mandate, achieving significant advances worldwide in the development of sciencebased animal welfare standards for food producing animals but also animals used in research and education, working equids, dog populations and farmed fish among others (OIE, 2021a,b). These OIE standards have been used to guide implementation of animal welfare standards in the public and private sectors (ISO, 2016).

Finally, the importance of social sciences (psychology, sociology, ethics) and community engagement are often determinant in the success of translating scientific findings into applications and solutions in practice (Lund et al., 2006; Miele et al., 2011; Sonntag, 2018). Given that animal welfare is constantly evolving according to our scientific knowledge of the needs of animals, and in the way humans influence animal welfare, it is important to continuously monitor and review the processes and conditions guiding animal welfare in practice. This requires seeking new perspectives and new ways of viewing challenges, in order to meet the evolving demands of society and incorporate positive welfare for a balanced approach to animal welfare.

\section{DISCUSSION AND CONCLUSIONS}

There has been a development originating in high-income Western countries to consider animal welfare, in the way that most people use this term at present. At first, this development underpinned by the emerging animal welfare science focussed on avoiding animal welfare challenges, i.e., "negative welfare". In the past two or three decades, however, there has been a shift in the same countries to consider so-called positive welfare where the focus is on behavioural expression and being positively engaged, with an on-going discussion about the underlying idea of what constitutes a good animal life. Animal welfare science is only beginning to catch up with this development but there are some potential dilemmas between limiting welfare challenges and increasing opportunities to allow behavioural expression and being positively engaged.

In this paper, we have considered the opportunities and challenges arising from broadening this development to include low-income countries. We argued that the shift toward positive welfare is not only a potential burden but also an opportunity for low-income countries. In high-income countries, intensive housing is more common, with animals sometimes lacking opportunities for behavioural expression or being positively engaged. Conversely, in low-income countries, it is often more about the provision of resources to the animals to prevent animal welfare challenges. There are potential economic incentives to improve welfare in a way where positive welfare and avoidance of welfare challenges go hand in hand. There are also opportunities for local cultural transformations where positive animal welfare becomes part of the local mind-set, with the opportunity for local developments of the idea of a good animal life.

There is however, currently a lack of data on the extent to which different animals kept in low-income countries, such as free-roaming livestock and dogs, experience more positive welfare and how well welfare challenges are being controlled. Animal welfare assessment and benchmark measures should be developed and taken up through engagement with stakeholders in various parts of the world. 


\section{DATA AVAILABILITY STATEMENT}

The

further inquiries

corresponding author. contributions

included

can be in

in the article, directed to the

\section{AUTHOR CONTRIBUTIONS}

J-LR initiated the idea. J-LR, PS, QS, and LS collaborated in writing a series of drafts of the manuscript. PS drafted Box 1. QS drafted Box 2. All authors contributed to the article and approved the submitted version.

\section{REFERENCES}

Amat, M., Camps, T., Brech, S., and Manteca, X. (2014). Separation anxiety in dogs: the implications of predictability and contextual fear for behavioural treatment. Animal Welfare 23, 263-266. doi: 10.7120/09627286.23.3.263

Amauchi, J., Gauthier, M., Ghezeljeh, A., Giatti, L., Keats, K., Sholanke, D., et al. (2021). The power of community-based participatory research: ethical and effective ways of researching. Commun Dev J. doi: 10.1080/15575330.2021.1936102

Boissy, A., Manteuffel, G., Jensen, M., Moe, R., Spruijt, B., Keeling, L., et al. (2007). Assessment of positive emotions in animals to improve their welfare. Physiol. Behav. 92, 375-397. doi: 10.1016/j.physbeh.2007.02.003

Braae, U. C., Penrith, M.-L., Ngowi, H. A., Lekule, F., and Johansen, M. V. (2016). Awareness concerning optimal pig production management and animal welfare among smallholder farmers in Tanzania. Animal Welfare 25, 439-446. doi: 10.7120/09627286.25.4.439

British Farm Animal Welfare Council (1993). Second Report on Priorities for Research and Development in Farm Animal Welfare. British Ministry of Agriculture, Fisheries and Food.

Broom, D. (1991). Animal welfare: concepts and measurement. J. Animal Sci. 69, 4167-4175. doi: 10.2527/1991.69104167x

Butterworth, A., Mench, J., Wielebnowski, N., and Olsson, A. (2018). Practical Strategies to assess (and improve) Welfare, in animal welfare, eds M. C. Appleby, I. S. Olsson, and F. Galindo (Wallingford: CAB International), 232-250. doi: 10.1079/9781786390202.0232

Carnovale, F., Jin, X., Arney, D., Descovich, K., Guo, W., Shi, B., et al. (2021). Chinese public attitudes towards, and knowledge of, animal welfare. Animals 11:855. doi: 10.3390/ani11030855

Dawkins, M. (2008). Animal suffering: the science of animal welfare. Ethology 114, 937-945. doi: 10.1111/j.1439-0310.2008.01557.x

Dawkins, M. S. (2021). The Science of Animal Welfare: Understanding What Animals Want. Oxford: Oxford University Press.

eSandoe, P., and Jensen, K.K. (2013). “The idea of animal welfare - developments and tensions," in Veterinary \& Animal Ethics: Proceedings of the First International Conference on Veterinary and Animal Ethics, 1st Edn, eds C. M. Wathes, S. A. Corr, S. May, S. McCulloch, and M. Whiting (Hoboken, NJ: Blackwell Publishing), 19-31. doi: 10.1002/9781118384282.ch2

Etiyibo, E. (2017). Anthropocentrism, african metaphysical worldview, and animal practices: A reply to kai horsthemke. J. Anim. Ethics. 7, 145-162. doi: 10.5406/janimalethics.7.2.0145

Fife-Cook, I., and Franks, B. (2019). Positive welfare for fishes: rationale and areas for future study. Fishes 4:31. doi: 10.3390/fishes4020031

Fraser, D. (2008). Understanding Animal Welfare: The Science in Its Cultural Context. Chicester: Wiley-Blackwell.

Grace, D., Mutua, F., Ochungo, P., Kruska, R., Jones, K., Brierley, L., et al. (2012). Mapping of Poverty and Likely Zoonoses Hotspots., in: Zoonoses Project 4. Report to the UK Department for International Development (Nairobi, Kenya: ILRI).

Harrison, R. (1964). Animal Machines: The New Factory Farming Industry. Wallingford, UK: CABI Publishing.

Heerwagen, L. R., Mørkbak, M. R., Denver, S., Sandøe, P., and Christensen, T. (2015). The role of quality labels in market-driven animal welfare. J. Agricult. Environ. Ethics 28, 67-84. doi: 10.1007/s10806-014-9521-z

Hemsworth, P. H. (2018). Key determinants of pig welfare: implications of animal management and housing design on livestock welfare. Animal Produc. Sci. 58, 1375-1386. doi: 10.1071/AN17897

International Fund for Agricultural Development (2013). Smallholders, Food Security, and the Environment. International Fund for Agricultural Development.

ISO (2016). (International Standards Organization). Iso/Ts 34700:2016 Animal Welfare Management-General Requirements and Guidance for Organizations in the Food Supply Chain, in: Technical Specification. Available online at: https:// www.iso.org/standard/64749.html

Keeling, L. J., Winckler, C., Hintze, S., and Forkman, B. (2021). Towards a positive welfare protocol for cattle: a critical review of indicators and suggestion of how we might proceed. Front. Anim. Sci. 2:753080. doi: 10.3389/fanim.2021.753080

Lassen, J., Sandøe, P., and Forkman, B. (2006). Happy pigs are dirty! Conflicting perspectives on animal welfare. Livestock Sci. 103, 221-230. doi: 10.1016/j.livsci.2006.05.008

Lawrence, A. B., Vigors, B., and Sandøe, P. (2019). What is so positive about positive animal welfare? - a critical review of the literature. Animals 9:783. doi: 10.3390/ani9100783

Lay, D. C., Fulton, R. M., Hester, P. Y., Karcher, D. M., Kjaer, J. B., Mench, J. A., et al. (2011). Hen welfare in different housing systems. Poultry Sci. 90, 278-294. doi: $10.3382 /$ ps.2010-00962

Lord, K., Feinstein, M., Smith, B., and Coppinger, R. (2013). Variation in reproductive traits of members of the genus canis with special attention to the domestic dog (Canis Familiaris). Behav. Processes 92, 131-142. doi: 10.1016/j.beproc.2012.10.009

Lund, V., Coleman, G., Gunnarsson, S., Appleby, M. C., and Karkinen, K. (2006). Animal welfare science-working at the interface between the natural and social sciences. Appl. Anim. Behav. Sci. 97, 37-49. doi: 10.1016/j.applanim.2005.11.017

McInerney, J. (2004). Animal Welfare, Economics and Policy. Report to the UK Farm and Animal Health Economics Division of Defra. Available online at: https://webarchive.nationalarchives.gov.uk/ukgwa/20130402151656/http:// archive.defra.gov.uk/evidence/economics/foodfarm/reports/documents/ animalwelfare.pdf

Mellor, D. J. (2015). Enhancing Animal Welfare by Creating Opportunities for Positive Affective Engagement. N. Zealand Vet. J. 63, 3-8. doi: 10.1080/00480169.2014.926799

Miele, M., Veissier, I., Evans, A., and Botreau, R. (2011). Animal welfare: establishing a dialogue between science and society. Animal welfare 20, 103-117.

Ndou, S., Muchenje, V., and Chimonyo, M. (2011). Animal welfare in multipurpose cattle production systems and its implications on beef quality. African J. Biotech. 10, 1049-1064.

Nkondo, G. (2007). Ubuntu as public policy in South Africa: a conceptual framework. Int. J. Afr. Renaissance Stud. 2, 88-100. doi: 10.1080/18186870701384202

OIE (2018). (World Organization for Animal Health). Chapter 7.1.-Introduction to the Recommendations for Animal Welfare, in: Animal welfare. (Paris: OIE).

OIE (2021a). (World Organization for Animal Health). Terrestrial Animal Health Code. Available online at: https://www.oie.int/en/what-we-do/ standards/codes-and-manuals/terrestrial- code- online-access/?id=169andL= landhtmfile=titre_1.7.htm/ (accessed Jan 21, 2022).

OIE (2021b). (World Organization for Animal Health). Aquatic Animal Health Code. Available: https://www.oie.int/en/what-we-do/standards/codes-andmanuals/aquatic- code- online-access/?id=169andL=1andhtmfile=titre_1.7. htm/ (accessed Jan 21, 2022).

OIE (World Organization for Animal Health) (2017). Animal Welfare Available online at: https://www.oie.int/en/what-we-do/animal-health-andwelfare/animal-welfare/ (accessed Nov 26, 2021).

Olsson, A. S., Nicol, C. J., Niemi, S. M., and Sandøe, P. (2019). From unpleasant to unbearable-why and how to implement an upper limit to pain and other forms of suffering in research with animals. ILAR J. 60, 404-414. doi: 10.1093/ilar/ilz018 
Paul, M., Majumder, S., Sau, S., Nandi, A., and Bhadra, A. (2016). High early life mortality in free- ranging dogs is largely influenced by humans. Sci. Rep. 6:19641. doi: 10.1038/srep19641

Perry, B., Robinson, T., and Grace, D. (2018). Review: animal health and sustainable global livestock systems. Animal 12, 1-10. doi: 10.1017/S1751731118000630

Pinillos, R. G. (2018). One Welfare: A Framework to Improve Animal Welfare and Human Well-Being. Wallingford: CABI Publishing. p. 112 doi: $10.1079 / 9781786393845.0000$

Price, E.O. (1999). Behavioral development in animals undergoing domestication. Appl. Anim. Behav. Sci. 65, 245-271.

Proops, L., Haddy, E., Brown, J., Zappi, H., Rodrigues, J.B., and Burden, F. (2021). "Exploring the relationship between owner attitudes and working equid welfare in Mexico, Portugal and Spain," in Third One Welfare World Conference 2021 (Burgos).

Qekwana, D., Mccrindle, C. M. E., Cenci-Goga, B., and Grace, D. (2019). “Animal welfare in Africa: Strength of cultural traditions, challenges and perspectives," in Animal Welfare: From Science to Law, eds. S. Hild and L. Schweitzer (Paris: La Fondation Droit Animal, Éthique et Sciences ), 103-107.

Rault, J.-L., Hintze, S., Camerlink, I., and Yee, J. (2020). Positive Welfare and the Like: Distinct Views and a Proposed Framework. Front. Vet. Sci. 7:370. doi: 10.3389/fvets.2020.00370

Sandøe, P., Hansen, H., Rhode, H., Houe, H., Palmer, C., Forkman, B., et al. (2020). Benchmarking farm animal welfare-a novel tool for cross-country comparison applied to pig production and pork consumption. Animals 10:955. doi: 10.3390/ani10060955

Seligman, M., and Csikszentmihalyi, M. (2000). Positive psychology: an introduction. Am. Psychol. 55, 5-14. doi: 10.1037/0003-066 X.55.1.5

Sonntag, I. (2018). Utilising Participatory Reflection and Action to Facilitate Rabies Control in a Rural Community in South Africa. Master thesis, University of Pre toria.

Spinka, M. (2019). Animal agency, animal awareness and animal welfare. Anim. Welfare 28, 11-20. doi: 10.7120/09627286.2 8.1 .011

Spooner, J., Schuppli, C., and Fraser, D. (2014). Attitudes of Canadian citizens toward farm animal welfare: a qualitative study. Livestock Sci. 163, 150-158. doi: 10.1016/j.livsci.2014.02.011
Vigors, B., Sandøe, P., and Lawrence, A. (2021). Positive welfare in science and society: differences, similarities and synergies. Front. Animal Sci. 2:738193. doi: 10.3389/fanim.2021.738193

Webb, L. E., Veenhoven, R., Harfeld, J. L., and Jensen, M. B. (2019). What is animal happiness? Annals N. Y. 1438, 62-76. doi: 10.1111/nyas.13983

Weeks, C., Lambton, S., and Williams, A. (2016). Implications for welfare, productivity and sustainability of the variation in reported levels of mortality for laying hen flocks kept in different housing systems: a meta-analysis of ten studies. PLoS ONE 11:e0146394. doi: 10.1371/journal.pone.0146394

Whiting, L., Kendall, S., and Wills, W. (2012). An asset-based approach: an alternative health promotion strategy? Community Practitioner. 85, 25-28.

Yeates, J. W., and Main, D. C. J. (2008). Assessment of positive welfare: a review. Vet. J. 175, 293-300. doi: 10.1016/j.tvjl.2007.05.009

Zambia Ministry of Fisheries and Livestock (2019). The 2017/2018 Livestock and Aquaculture Census Report, Ministry of Fisheries and Livestock, Zambia Statistics Agency, Lusaka. Available at: https:// www.zamstats.gov.zm/phocadownload/Agriculture/The\%202017-18 \%20Livestock\%20and\%20Aquaculture\%20Census\%20Summary\%20Report. pdf (accessed 21.01.2022).

Conflict of Interest: The authors declare that the research was conducted in the absence of any commercial or financial relationships that could be construed as a potential conflict of interest.

Publisher's Note: All claims expressed in this article are solely those of the authors and do not necessarily represent those of their affiliated organizations, or those of the publisher, the editors and the reviewers. Any product that may be evaluated in this article, or claim that may be made by its manufacturer, is not guaranteed or endorsed by the publisher.

Copyright (C) 2022 Rault, Sandøe, Sonntag and Stuardo. This is an open-access article distributed under the terms of the Creative Commons Attribution License (CC BY). The use, distribution or reproduction in other forums is permitted, provided the original author(s) and the copyright owner(s) are credited and that the original publication in this journal is cited, in accordance with accepted academic practice. No use, distribution or reproduction is permitted which does not comply with these terms. 